\section{Antibiotics for mastitis in breastfeeding women}

This is the abstract of a Cochrane Review published in the Cochrane Database of Systematic Reviews 2013, issue 2, art. no. CD005458. DOI: 10.1002/14651858.CD005458.pub3. For full text and details about the authors, see reference 1.

\section{Shayesteh Jahanfar, Chirk Jenn Ng, Cheong Lieng Teng \\ The independent commentary was written by César Eduardo Fernandes}

\section{ABSTRACT}

BACKGROUND: Mastitis can be caused by ineffective positioning of the baby at the breast or restricted feeding. Infective mastitis is commonly caused by Staphylococcus aureus. The prevalence of mastitis in breastfeeding women may reach 33\%. Effective milk removal, pain medication and antibiotic therapy have been the mainstays of treatment. OBJECTIVES: This review aims to examine the effectiveness of antibiotic therapies in relieving symptoms for breastfeeding women with mastitis with or without laboratory investigation.

\section{METHODS:}

Search methods: We searched the Cochrane Pregnancy and Childbirth Group's Trials Register (30 September 2012), contacted investigators and other content experts known to us for unpublished trials and scanned the reference lists of retrieved articles.

Selection criteria: We selected randomised controlled trials (RCTs) and quasi-RCTs comparing the effectiveness of various types of antibiotic therapies or antibiotic therapy versus alternative therapies for the treatment of mastitis.

Data collection and analysis: Two review authors independently assessed trial quality and extracted data. When in dispute, we consulted a third author.

MAIN RESULTS: Two trials met the inclusion criteria. One small trial $(n=25)$ compared amoxicillin with cephradine and found no significant difference between the two antibiotics in terms of symptom relief and abscess formation. Another, older study compared breast emptying alone as 'supportive therapy'versus antibiotic therapy plus supportive therapy, and no therapy. The findings of the latter study suggested faster clearance of symptoms for women using antibiotics, although the study design was problematic.

AUTHORS CONCLUSIONS: There is insufficient evidence to confirm or refute the effectiveness of antibiotic therapy for the treatment of lactational mastitis. There is an urgent need to conduct high-quality, doubleblinded RCTs to determine whether antibiotics should be used in this common postpartum condition.

The full text of this review is available free-of-charge from: http://onlinelibrary.wiley.com/doi/10.1002/14651858.CD005458.pub3/epdf

The abstract is also available in the Portuguese and English languages

\section{REFERENCE}

1. Jahanfar S, Ng CJ, Teng CL. Antibiotics for mastitis in breastfeeding women. Cochrane Database Syst Rev. 2013;2:CD005458.

\section{COMMENTS}

The present review addresses an important issue involving the use of antibiotics to treat mastitis in breastfeeding women, which is common in its infectious form. Considering its infectious nature, the treatment has traditionally consisted of using antibiotics. However, the basis of the present knowledge is empirical and this topic deserves a review.

Only two studies met the inclusion criteria proposed for this review. One, which comprised a small sample, compared two antibiotics, amoxicillin and cephradine, and showed no difference between them in terms of relieving the symptoms and abscess formation. Another study, in 1984, conducted with a larger sample and with no description of blinding methods, monitored breastfeeding women with infectious mastitis divided into three groups: one receiving no treatment; another advised to empty the breasts; and the third treated with antibiotics (penicillin, ampicillin or erythromycin). This study found that the symptoms improved more rapidly among the women who used antibiotics. Based on the studies analyzed, the authors of the review concluded that there was insufficient evidence to confirm or refute the efficacy of antibiotic therapy for treating mastitis and urgently recommended that appropriate studies should be conducted to seek answers. From the conclusion of this Cochrane review and the current knowledge, we believe that the decision to use antibiotics in cases of infectious lactation mastitis remains eminently clinical and the final decision regarding this matter should be made by the physician.

César Eduardo Fernandes. Full Professor of Gynecology, Faculdade de Medicina do ABC (FMABC), Santo André, SP, Brazil. 